\title{
The Impact of the Covid-19 Cases with Twitter Users in Their Perception of the Brazilian Government
}

\author{
Celso de Oliveira Pacheco Junior \\ Federal University of the \\ State of Rio de Janeiro (UNIRIO),
Rio de Janeiro, RJ, Brazil
celsojunior@edu.unirio.br

Abstract

During the COVID-19 pandemic, political discussions in Brazil revolved around the pandemic and the controversial leader Jair Bolsonaro. Twitter reflected these discussions, also bringing reports of user dramas that had family members victimized by Covid-19. This study investigates their perception of the federal government through a quantitative and qualitative analysis of these users' tweets. We have identified 3,756 Twitter users who reported cases of family members with Covid-19 and collected their government-related tweets before and after those reports. We analyzed the feelings expressed in these tweets using automated techniques and extracted a sample of 650 tweets that had difficult-to-understand terms for a manual analysis of the feelings. The study found subtle changes in the perceptions of people who approve or disapprove of the federal government.

\section{Introduction}

Social media has had a major impact on public discourse and society communication in the last decade. In this scenario, Twitter has emerged as one of the most dominant platforms in the area of political discourse [1] [2] [3]. While the 280-character limit seems restrictive, Twitter posts reveal enormous reach and influence on the political landscape around the world [4] [5]. An important feature of Twitter is its real-time nature that allows its data to be captured to predict election results [6] [7], identify political ties on social media [8] [9] and identify communication patterns, besides interactions related to political events [10] [11].

Over the past two years, political discussions on Twitter in Brazil have revolved around two themes: i) the Covid-19 pandemic, which collapsed the Brazilian healthcare system and impacted on country economy; and ii) the Jair Bolsonaro government, which has a legion of over 6 million followers and frequently uses the platform to publicize government actions, post and

\author{
Sean Wolfgand Matsui Siqueira \\ Federal University of the \\ State of Rio de Janeiro (UNIRIO), \\ Rio de Janeiro, RJ, Brazil \\ sean@uniriotec.br
}

retweet messages against opponents who are on the opposite ideological spectrum.

In May 2021, a CPI (Parliamentary Commission of Inquiry) was created to investigate responsibility for the health tragedy in Brazil. One of the aims of this commission is to investigate omissions in the fight against the pandemic by the federal government. The moment of installation of this parliamentary commission coincides with the worst levels of approval of the president of Brazil and with the increase in the role of ex-president Luís Inácio Lula da Silva. Recently, Lula regained his political rights after a long legal battle and surpassed Bolsonaro in all polls of voting intentions for the general elections that will take place in 2022[12].

The pandemic is at the center of political debate at the CPI and also on social media. Twitter has been an important stage for the press, government supporters and detractors to express their views on government actions in managing the health crisis. In addition, we realize that many people also use the platform to report personal or family stories affected by Covid-19. An analysis of feelings about these tweets would allow us to understand whether the family dramas provoked by Covid-19 have altered the perception of Twitter users towards the federal government.

This study used quantitative and qualitative analysis methods to investigate whether Twitter users who reported family dramas caused by Covid-19 changed their perceptions concerning the Jair Bolsonaro government. Posts referring to the Jair Bolsonaro government were collected and organized in two datasets: before and after the users first reported a pain concerning close victims of Covid-19.

An analysis of the users' feelings in these two datasets was carried out and compared based on metrics such as the average of likes in the period, average of tweets in the period, intensity analysis, word cloud, polarity and subjectivity in both data groups.

After applying the analysis of feelings in the posts, this study identified that the general feeling of people towards the Brazilian government fluctuated, on 
average, from neutral to weak negative. Tweets with anti-government messages expressed negative feelings such as death, pandemic and loss. It was also noticed the concern about hunger.

This paper is organized in six sections including this introduction. In section 2, it is introduced the works related to the theme. Section 3 presents the methodology used to collect tweets, preparing and analyzing the sentiments expressed in the groups of collected tweets. In section 4, the results of the sentiment analysis metrics performed in this study are presented. In section 5, the findings of our research are discussed regarding the literature. Finally, section 6 presents the conclusions of this paper.

\section{Literature Background}

Sentiment analysis is a powerful approach and it is at the heart of social media research. A set of natural language processing techniques can be used to analyze a person's opinions [13], extract lexical feelings and classify emotions as positive, negative or neutral [14] [15] [16].

In the last year, the Covid-19 pandemic has impacted the lives of people all over the world. Therefore, studies are underway to look at sentiments about the new coronavirus from many perspectives, and conclusions are drawn from a wide range of available tools and techniques [17] [13].

Dubey et al. [18] collected tweets for 20 days in March 2020. Data from four continental European states were collected to analyze people's sentiments during the explosion of the new coronavirus. Their study found out that many people worldwide experienced feelings of fear, sorrow and disgust, although most people on the planet were taking a positive and hopeful approach.

Medford et al. [19] applied unsupervised machine learning techniques to analyze the data collected on the coronavirus. Their study aims to quantify and understand the sentiments of Twitter users about the Covid-19 pandemic. 126,049 tweets were evaluated, and almost half expressed fear.

Garcia et al. [20] ranked ten topics related to the Covid-19 pandemic and analyzed the content discussed on Twitter for four months. The study analyzed the evolution of people's speech and behavior over time in Brazil and the United States. The topics they identified were representative of the media during April and August 2020 in both countries. Their study analyzed sentiment trends over a long period, related them to the news reported, and compared human behavior in two different geographic locations affected by the pandemic.

Other studies have used sentiment analysis techniques and tools to understand people's feelings toward a political group. Kaur et al. [21] explored how emotions are portrayed by leaders on Twitter, particularly during crises like Covid-19. Their study also addresses a need to establish ties of trust with authorities for the gradual dissemination of knowledge to the public. A total of 12,128 tweets were extracted from 29 Indian political leaders from the day of the Covid-19 pandemic declaration to Lockdown 2.0. The results show Anger, Anticipation, Disgust, Fear, Joy, Sadness, Surprise, Confidence, Negative and Positive sentiments. However, Positive and Confidence sentiments were the most frequent whenever authorities issued or communicated the new guidelines.

Iyad \& Dahrooj [22] presented an analytical study to measure political public opinion regarding the Palestinian-Israeli conflict using Twitter data. The study uses a new data analysis model that leverages two levels of analysis: country-level analysis and individual-level analysis. The country-level analysis explored the country's overall attitude toward Palestine, identifying the counties which generated the most topic-focused tweets, measuring each country's sympathy for Palestine, and analyzing the shift in sentiment over time. The individual-level analysis explored the activity and background of individuals. As a result, this study can provide informative content for other developers and data analysts interested in analyzing social media sentiment about political conflicts in particular.

Despite presenting studies that analyze sentiments in the context of the Covid-19 and government pandemics, it was not identified a related work that explores possible changes in users' perception of the government, especially comparing before and after reporting personal dramas and family tragedies caused by Covid-19 on social media.

\section{Methodology}

This section presents an overview of the steps performed in this research. In this study, we used a quanti-qualitative analysis to classify Twitter messages.

Firstly, an extraction was made of users who posted reports of family members victimized by Covid- 19 . The corresponding tweet was marked as a base tweet. There are cases where users have posted multiple tweets. In these cases, the tweet with the oldest date was considered as the base tweet. The Tweepy library [23] of the Python language was used to extract tweets. In the process of identifying the base tweets, we searched for all tweets containing the terms: ("my father" OR "my mother" OR "my son" OR "my daughter" OR "my 
uncle" OR "my aunt" OR "my grandfather" OR "my grandmother" OR "my brother" OR "my sister" OR "my cousin" OR "my nephew" OR "my niece") AND (died OR "passed away" OR had OR got OR hospitalized OR cured OR healed OR "gotbetter" OR discharged) AND (corona OR coronavirus OR "corona virus" OR covid OR covid-19) ${ }^{1}$. The data extraction period took place in 72 consecutive days of data collection between $03 / 30 / 2021$ and 06/10/2021, and 3,756 unique users were identified. For each of the 3,756 users, we collected tweets before and after their respective base tweet, as shown in Figure 1.

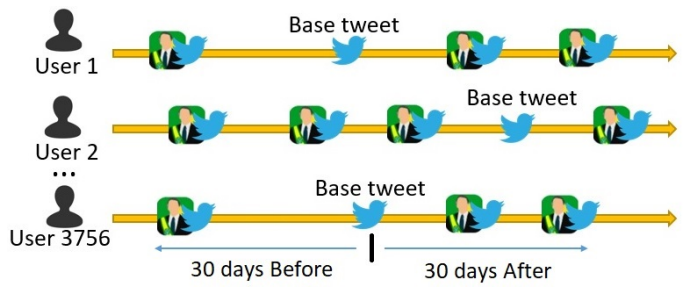

Figure 1. Base tweets and the before and after grouping for analysis

In order to obtain a minimum amount of tweets before and after the base tweet, it was necessary to restrict the collection period. We defined a time window for this second tweet extraction phase of 30 days before and 30 days after each user's base tweet. The choice for the 30-day interval is based on studies showing that the hospitalization period at Covid-19 is an average of 22 days [24]. Then we applied a filter to select only tweets about the Bolsonaro government. As a result of applying this last filter, we obtained two sets of tweets about the Bolsonaro government, one before the base tweet and one after it. We use these two sets of tweets to perform our analysis.

The quantitative approach followed the inductive research method using the calculation of sentiment analysis metrics, number of tweets, likes, retweets, and word frequency to investigate the possible change in users' perception about the Bolsonaro government. We used the VADER model (Valence Aware Dictionary and Sentiment Reasoner) [25] to analyze the sentiments' intensity. This tool performs sentiment analysis based on lexical characteristics and rules to evaluate specific texts from social networks, such as emojis. Tweets about the Bolsonaro government had to be translated into English as the VADER model does not have a lexical dictionary in Portuguese. According to Pereira [26], translating texts into English and using tools

\footnotetext{
${ }^{1}$ To perform the search on twitter, the terms were used in Portuguese, but in this study they were written in English to facilitate the reader's understanding.
}

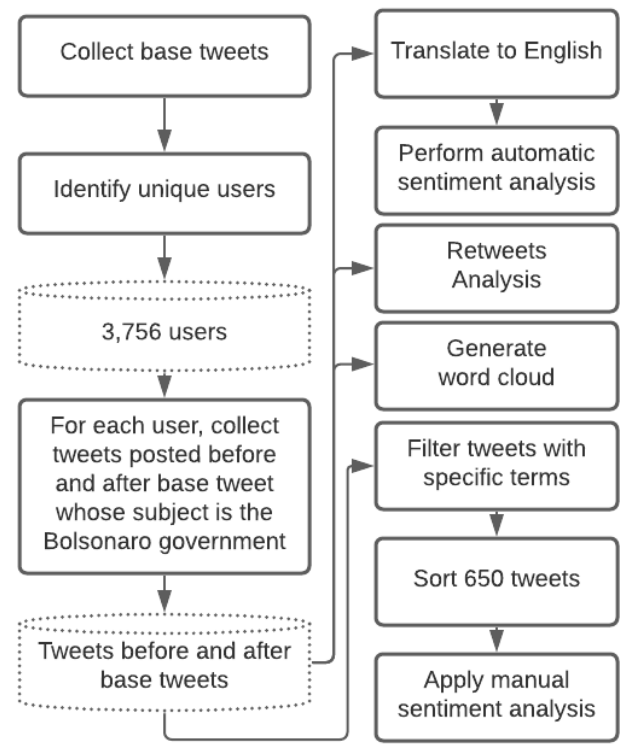

Figure 2. The tweets' analysis process

developed for that language is more effective than applying specific tools and efforts in Portuguese. We use the Google Translator API, a Python language translation library that supports the automatic translation of large volumes of text, to translate the tweets. The TextBlob library [27], developed in Python, was used for polarity and subjectivity analysis. Polarity indicates the intensity or strength of the emotion expressed through the degree of positivity and negativity of a text. To represent polarity, the TextBlob library uses values between -1.0 and +1.0 , where -1.0 refers to $100 \%$ negative polarity, +1.0 refers to positive polarity, and 0 indicates neutral polarity. Complimentarily, subjectivity indicates a personal opinion in a text [26]. The TextBlob library uses values between 0.0 and 1.0 to represent the subjectivity of a text, where 0.0 refers to a $100 \%$ objective value (facts or information) and 1.0 represents a $100 \%$ subjective value (opinions). We also implemented charts to make it easier to view and perform a comparative analysis of the data before and after the base tweet.

In the qualitative approach, we performed a manual classification of sentiments on a sample of tweets. In this approach, we filtered out relevant terms in the tweets that the translation tool did not understand or tweets which we suspect there was a misqualification by the sentiment analyzer. Of the two groups of tweets about the government, we used a sample of 650 tweets with the terms: CPI, Pfizer, Lula, Genocida (genocide), 29M (social movement on May 29th, 2021, 
against Bolsonaro), Impeachment and ForaBolsonaro (outBolsonaro). Like this, We developed and made an available web application ${ }^{2}$ (Figure 3 ) to allow users to rate tweets.

The participants were selected by convenience considering the authors' contacts: five professors, a psychologist, three computer system analysts, a geologist, a geophysicist, a lawyer, a journalist and a geographer. All selected ones are frequent social networks users and have completed at least higher education. Each user rated between 30 to 50 tweets. Tweets were classified as neutral, positive (strong and weak) or negative (strong and weak).

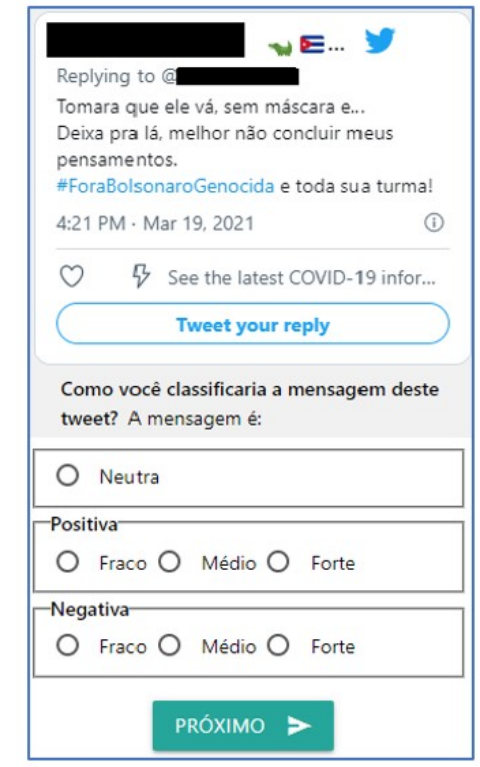

Figure 3. Artifact developed for manual classification of tweets

Table 1 shows the total amount of tweets about the Bolsonaro government before and after the base tweet in the analytic approaches.

Table 1. Groups of tweets and their amounts

\begin{tabular}{|c|c|c|}
\hline Analisys & Tweet Group & Quantity \\
\hline \multirow{2}{*}{ Automatic } & Before & 2,057 \\
\cline { 2 - 3 } & After & 10,607 \\
\hline \multirow{2}{*}{ Manual } & Before & 325 \\
\cline { 2 - 3 } & After & 325 \\
\hline
\end{tabular}

Thus, we matched the automatic (quantitative), and the manual (qualitative) sentiment analysis approaches.

\footnotetext{
${ }^{2}$ The manual classification dataset is available at: https://github.com/juniori/covid19_users_perception_qualitative_research
}

\section{Results}

In this work, the sentiment analysis considered a set of metrics that allowed us to assess whether or not there was a change in the perception of people on Twitter in relation to the Brazilian government after the report of beloved ones victimized by Covid-19 in Brazil. We considered the following metrics: intensity, average likes and retweets, polarity and subjectivity, and word cloud. The manual analysis allowed comparison with the results obtained by the automatic analysis.

\subsection{Calculation of Intensity Over the Period}

The VADER sentiment analyzer, described in section 3, was used to calculate sentiment intensity. This analyzer receives the tweet as input and generates the polarity of the entire sentence, analyzing it word by word. We applied a mechanism to classify sentiment into positive, neutral and negative [28] [29] [30] and its subcategories, as illustrated in Figure 4.

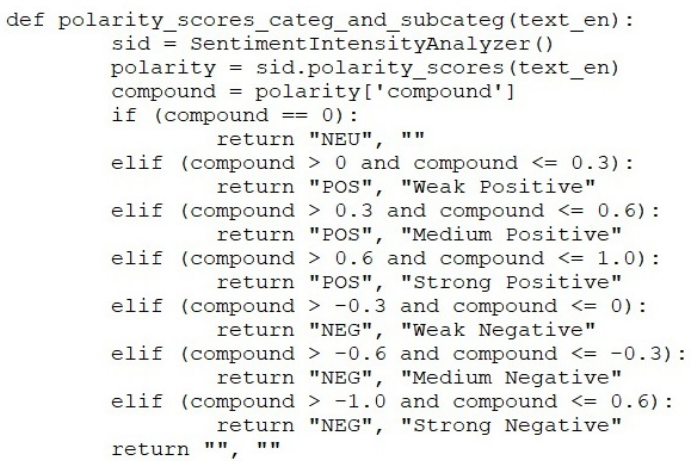

Figure 4. The method used to assign sentiment value to the text

Figure 5 shows the percentages of sentiment categories and subcategories referring to the sets of tweets related to the Brazilian government before and after the base tweet.

The compound score [25] is calculated by adding the valence scores of each lexical content, adjusted according to the rules, and then normalized to be between -1 , which represents the negative extreme, and +1 , which represents the positive extreme. A set of Scatter Plots is presented in Figure 6 to enrich this analysis. It shows the number of tweets and the respective average of compounds of users before and after each user's base tweet. 


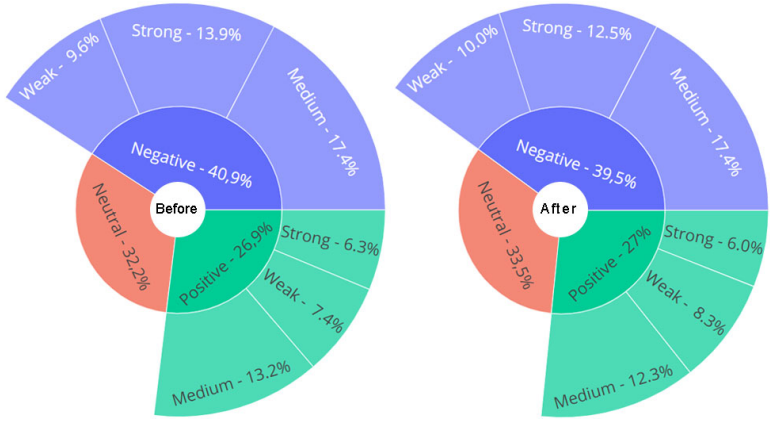

Figure 5. The Intensity of Sentiment Before and After the base tweet

\subsection{Average of Likes per Period}

In the digital engagement assessment, quantitative metrics are provided, such as the number of likes, shares, comments, opens, views, followers or clicks, as indicators of the level of engagement or valence of engagement (positive or negative comments) [31]. The greater the number of likes, the greater the similarity of thoughts between the interlocutor and other people in the social network. In this study, we grouped the data by date and ranked the intensity of government-related tweets. We added the number of likes for each tweet on a daily basis and calculated the average using appropriate functions to create a time-series graph of the average likes for the tweets rated as positive, neutral, and negative.

Figure 7 shows that over the time series, there is a greater amount of likes on April 9th and June 4th for negative tweets, April 10th for positive tweets, and April 11 th for neutral tweets.

\subsection{Average of Retweets by Period}

The average of retweets allows complementing the analysis of similarity of thought among people in the social network. The action of retweeting expresses people's consent to the tweet. People can also retweet tweets that were retweeted previously. In our study, we calculated the average of retweets as we did the average of likes and generated the graph in Figure 8 that shows the time series of the average of retweets. In this case, we observe that retweets are also the tweets most liked.

\subsection{Polarity and Subjectivity}

Polarity metrics are used to indicate the person's behavior through the intensity of the emotion in the tweet. On the other hand, subjectivity measures the user's opinion about the subject in the tweet. We used the TextBlob library function [27], which receives

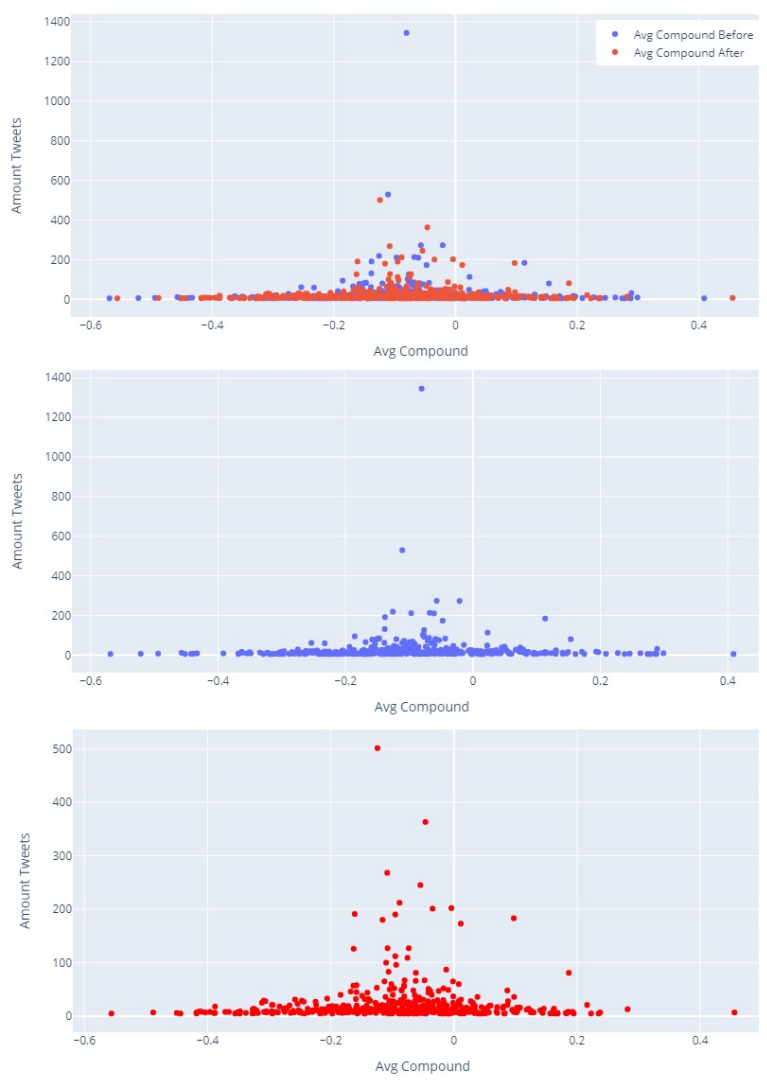

Figure 6. Compound vs. Amount of Tweets

the tweet and returns the measure of polarity and subjectivity. The average of these values was obtained for each day of the data collection period and grouped by tweets before and after the respective base tweets. The first base tweet was extracted on March 28th. From then on, tweets about the Brazilian government began to be extracted. Figure 9 shows the polarities of the tweet groups before and after the base tweets. Polarity score values range from $[-1$ to +1$]$. Thus, the value -1 represents negative sentiment, and +1 represents positive sentiment.

Figure 10 shows the subjectivities of the tweet groups before and after the base tweets. In this evaluation, the score values range from $[0$ to +1$]$, where 0 represents the text with some opinion and 1 represents the maximum degree of opinion.

\subsection{Word Cloud}

Previously, tweets were grouped into positive, neutral and negative categories. A word cloud is presented for each category, showing different words that often appear for the respective category before and after the base tweet. Figure 11 shows the word clouds 


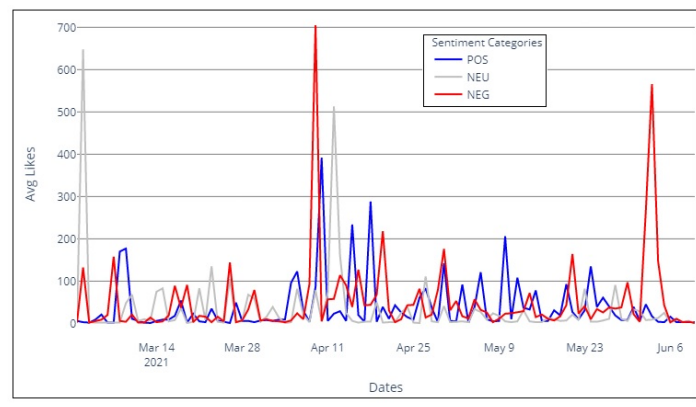

Figure 7. Average of Likes over the Period

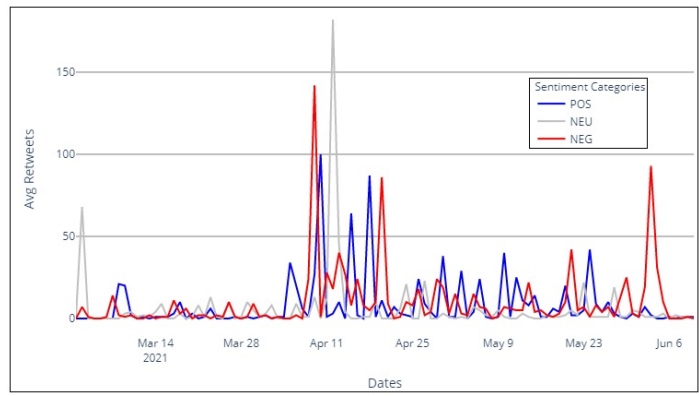

Figure 8. Average of Retweets over the Period

for tweets before their base tweets.

Figure 12 shows the word clouds for tweets after the respective base tweets. The bigger the size of the word, the greater its frequency of occurrence in texts. For creating our word cloud, we removed the irrelevant and non-emotional words.

\subsection{Manual Analysis}

From the terms considered for analysis, "Genocide" is the one that stood out the most, appearing in 192 tweets of the 650 evaluations, followed by the words "Lula" and "CPI" with respectively 137 and 127 tweets. The term "Genocide" was also the one with the greatest correspondence between the manual and automatic classifications, totaling a little more than $65 \%$ of correspondence. We noticed that the percentage of correspondence in manual and automatic classifications was higher for negative terms. The match for the negative sentiment of the term "Genocide" was $72 \%$. The term "Genocida" (genocide) was classified as neutral in the word cloud because of an error in the Google Translate API translation. We found a good match between manual and automatic classifications of tweets with the word "Genocide" due to the context of the phrase.
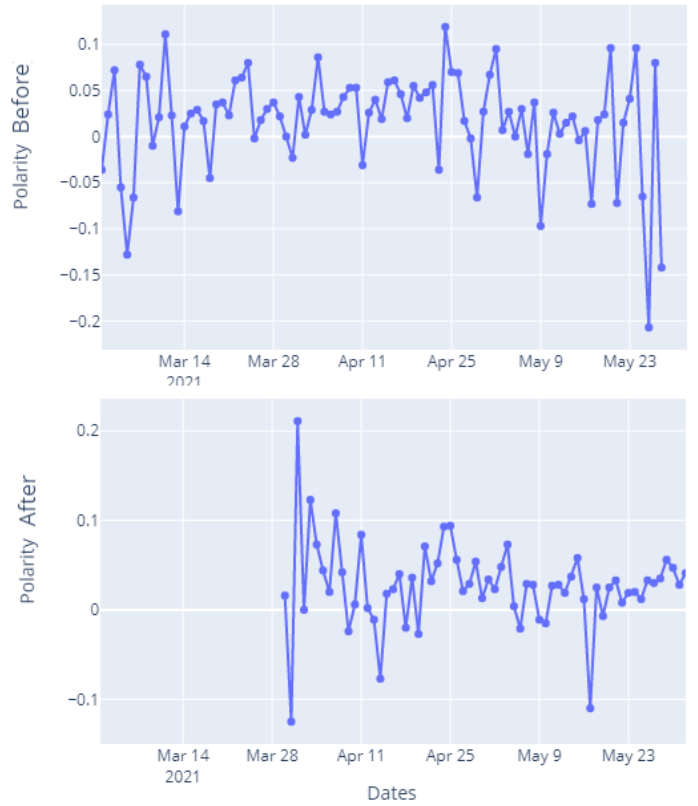

Figure 9. The calculated average of polarity over the period

\section{Discussion}

There is the figure of the "good citizen" [32] [33] in the scenario of political discussions in and out social networks. This figure is a Manichean representation in the ideological discourse of a portion of the population that claims for themselves the distinction of superiority in relation to groups already historically marginalized in (Brazilian) society. In the rhetoric of the "good citizen," only hetero-patriarchal families, Christians and defenders of family and property should have their constitutional rights preserved. The Bolsonaro government makes commercial and political use of the figure of the "good citizen". Based on the above concept, we created the search string of our filter to extract the tweets from the most conservative and historically ideologically aligned family nucleus to this government and check if there was a change of opinion. The intensity calculation, illustrated in Figure 5, does not suggest any significant change in the percentages of sentiment towards the Brazilian government before and after the base tweet. The collected data did not show significant differences in the percentages of categories or subcategories. However, the graphs shown in Figure 6 illustrate a higher density of users between the composite values -0.4 and 0.2 .

This might suggest that reporting tweets from people close to you may have increased people's involvement with tweets about the Brazilian government. People's engagement can be seen in the number of likes and 

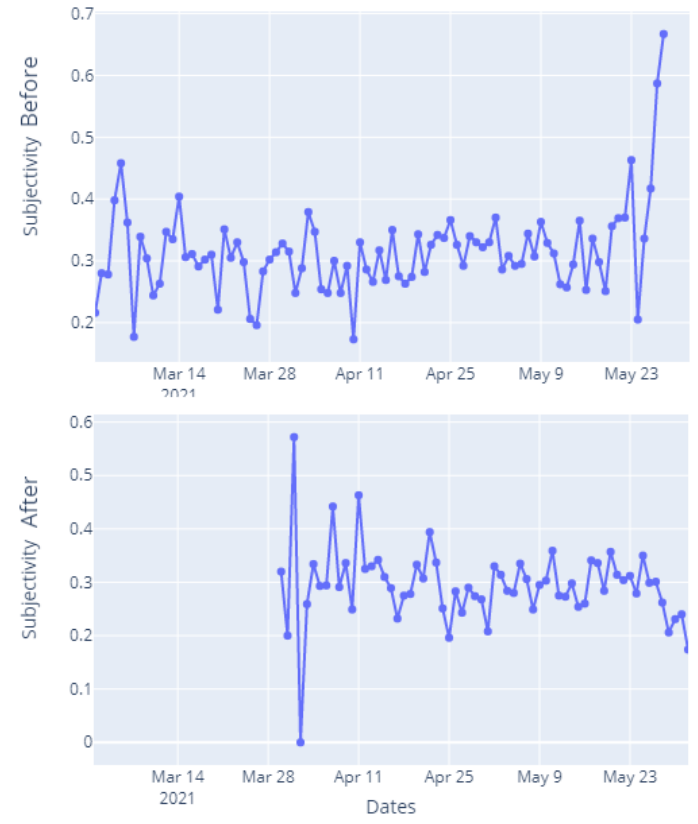

Figure 10. The calculated average of subjectivity over the period

retweets in the time series of positive, neutral and negative tweets about the Brazilian government. From these observations, the previous and aftermost groups were analyzed together, and we observed that there were spikes in likes that were immediately followed by spikes in retweets within a few days. Some tweets are catalysts of sentiments that leverage the average amount of retweets. For example, the 4-9 tweet, categorized as Strong Negative, represented by Figure 13, has 14,000 likes and over 2,300 retweets. There were few exceptions where spikes occurred, and the polarity and subjectivity metrics did not show any significant visible difference in the graphs before and after the base tweet. The polarity graphs showed daily averages with values normally orbiting in intervals of -0.1 to +0.1 . Subjectivity graphs also have daily averages concentrated in the ranges between 0.2 and 0.4 .

Most of the reported works [18] [19] [20] collected tweets about Covid-19 to analyze people's feelings directly about the disease. This study performed an indirect analysis because we used base tweets as time markers and grouped government-related tweets before and after base tweets. Therefore, unlike the works cited, the main objective was not to analyze people's feelings towards the pandemic, but rather the feeling towards the government in the face of this great tragedy that afflicts people. When comparing the word clouds of tweets about the Brazilian government before and after the base tweet, it was noticed the increase in
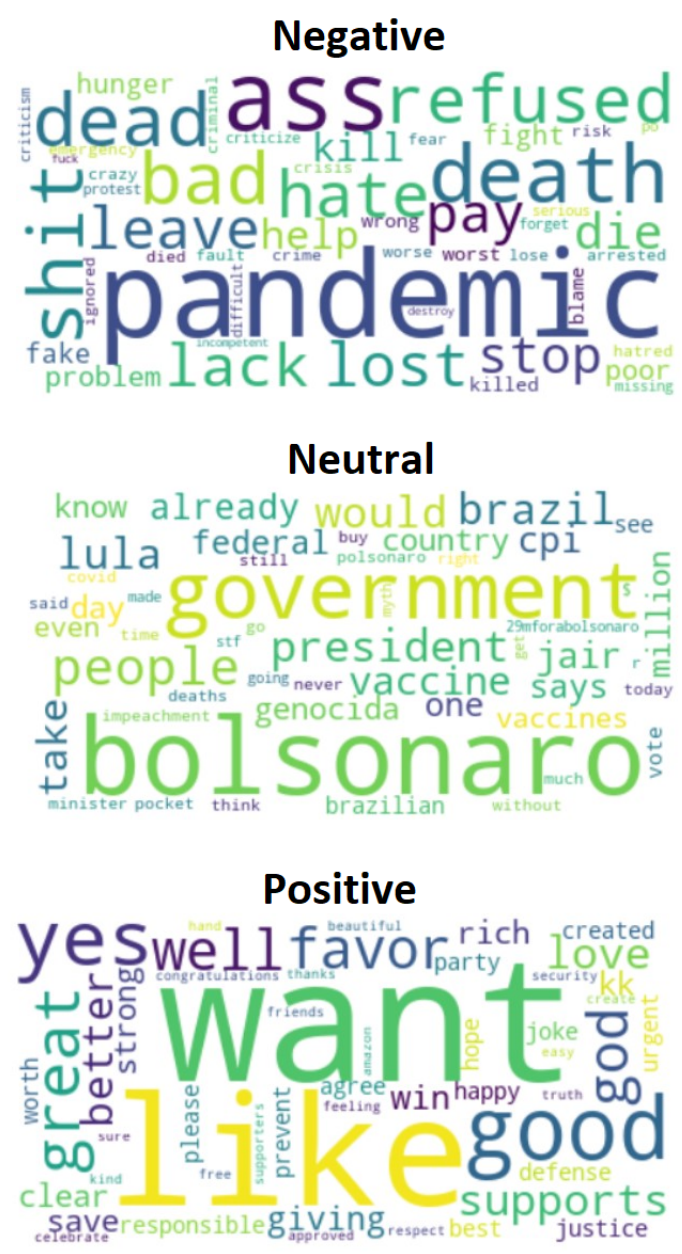

Figure 11. Word Clouds for positive, neutral and negative categories before the base tweet

the size of the words "pandemic", "protest", "hate", "death", "hunger" and "shit". These words can suggest the feelings of people who have experienced personal drama or trauma after a beloved one has been infected with the disease. The increase in the size of the word "hunger", for example, illustrates the growing concern about hunger during the pandemic. The National Survey on Food Insecurity in the context of the Covid-19 Pandemic in Brazil, carried out by Brazilian Research Network on Food and Nutritional Sovereignty and Security, indicates that in the last months of 2020, 19 million Brazilians were hungry and more than half of the families in the country faced some degree of feeding insecurity. A comparison between the word clouds of positive tweets before and after the base tweets showed that the words "want", "like" and "good" remained prominent with slight variation in size before and after the base tweet. We observed an increase in the size 


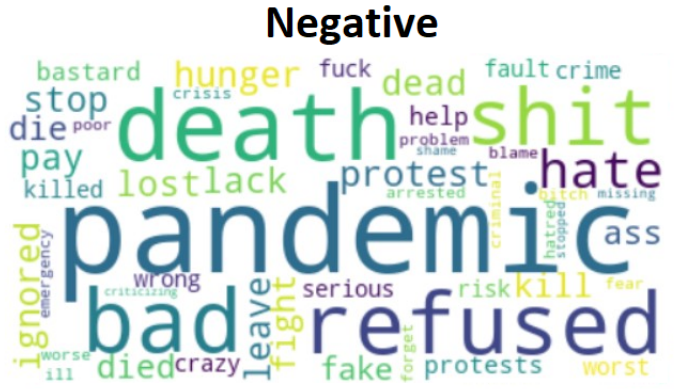

Neutral
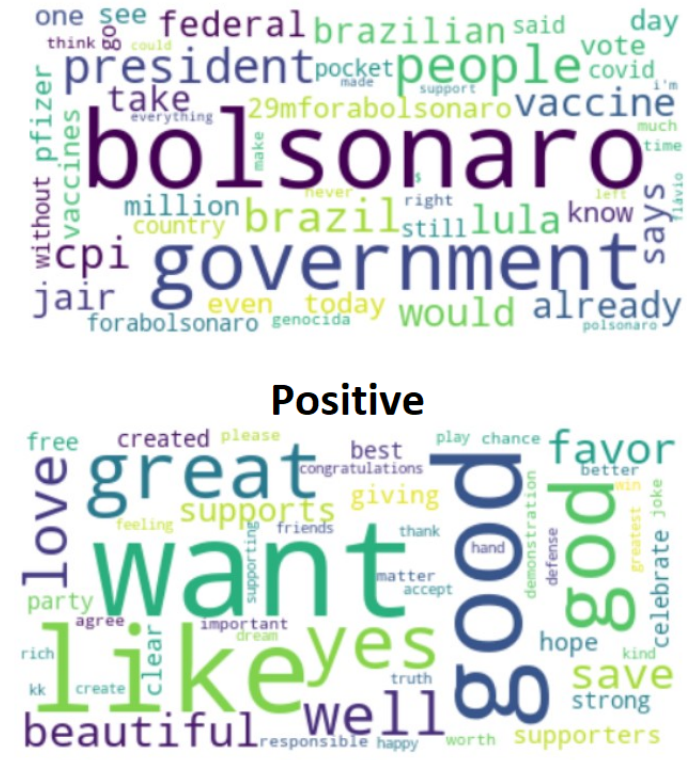

Figure 12. Word Clouds for positive, neutral and negative categories after the base tweet

of the word "God", which does not necessarily denote the president's strength with religious groups but may reinforce a position on a certain issue, such as faith in health recovery. The words "celebrate" and "party" also appear in the positive word cloud after the base tweet.

In the automatic analysis, it was identified that VADER's lexical dictionary does not have several negative terms related to the context of our research. Furthermore, VADER classifies words with a syntax error as neutral. Hence the importance of complementing the research with a manual analysis. However, many users reported difficulty in classifying sentences for the following reasons: i) they were emotionally involved with the topic and ii) they had difficulty understanding and classifying ironies and sarcasm.

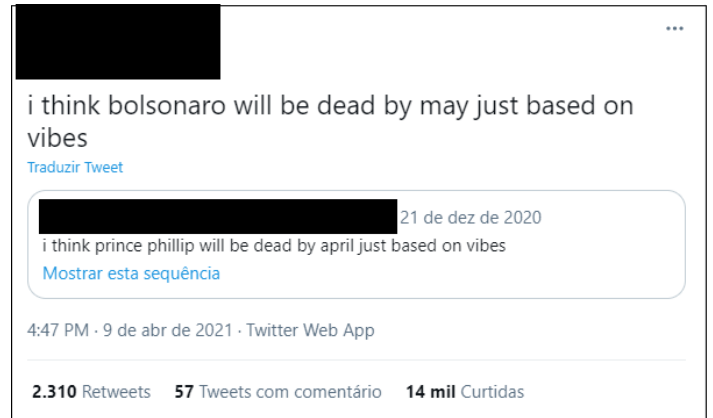

Figure 13. Example of tweet considered for analysis

\section{Conclusion}

Opinion polling on Twitter data has received much attention in recent years and involves dissecting the tweets and the content of these expressions [13]. The research presented in this paper used both qualitative and quantitative methods to analyze the sentiments of people related to the Brazilian government before and after posting reports from people close to them who suffered from Covid-19. We found that people's general sentiment towards the Brazilian government ranged, on average, from neutral to weak negative. However, in an analysis of the word clouds, there were subtle shifts in the concerns of the government supporters group and the anti-government group. Tweets with messages against the government expressed negative feelings such as death, pandemic and loss. In addition, new concerns emerged after the base tweet, such as those related to hunger. Through a manual analysis, it was possible to verify that although the term "Genocide" was not correctly translated by Google Translate, the context of the phrase allowed for a satisfactory correspondence with the automatic classification. Correspondences were higher for negative sentiments. We have also checked users' difficulty in sorting tweets manually.

This study has some limitations. The tweets collected for this study were in Portuguese and needed to be translated into English since the most effective approaches, such as the VADER model, do not have a lexical dictionary for the Portuguese language. The data collection period for users' tweets has also been restricted to a time window of 30 days before and 30 days after the base tweet. This time window can be expanded to investigate a possible difference in users' sentiments considering longer periods.

We plan to implement a sentiment rating using Bidirectional Transformer Encoding Representations (BERT) [34] along with logistic regression or another rating engine to understand better users' opinions of government before and after base tweets. In this study, 
we observed the groups of tweets before and after the base tweet, but we have not investigated whether each individual user changed his mind about the government after posting a report about someone close to Covid-19.

As future work, we intend to investigate how close the users were to their loved ones who had Covid-19 and how the severity of the disease impacted the users' feelings towards the Brazilian government.

The identification of base tweets was found on the family as biological relationships. We used this approach to bring tweets preferentially from conservative groups historically aligned with the Bolsonaro government. Then we assessed whether these groups changed their opinion regarding the government. However, as future work, we intend to include non-biological family backgrounds and compare the results.

\section{References}

[1] H. Kwak, C. Lee, H. Park, and S. Moon, "What is twitter, a social network or a news media?," in Proceedings of the 19th international conference on World wide web $W W W^{\prime} 10$, ACM Press, 2010.

[2] T. Sakaki, M. Okazaki, and Y. Matsuo, "Earthquake shakes twitter users," in Proceedings of the 19th international conference on World wide web - WWW'10, ACM Press, 2010.

[3] A. Java, X. Song, T. Finin, and B. Tseng, "Why we twitter," in Proceedings of the 9th WebKDD and 1st SNA-KDD 2007 workshop on Web mining and social network analysis - WebKDD/SNA-KDD '07, ACM Press, 2007.

[4] A. Bessi and E. Ferrara, "Social bots distort the 2016 u.s. presidential election online discussion," First Monday, Nov. 2016.

[5] H. Allcott and M. Gentzkow, "Social media and fake news in the 2016 election," Journal of Economic Perspectives, vol. 31, pp. 211-236, May 2017.

[6] A. Tumasjan, T. Sprenger, P. Sandner, and I. Welpe, "Predicting elections with twitter: What 140 characters reveal about political sentiment," Proceedings of the International AAAI Conference on Web and Social Media, vol. 4, May 2010.

[7] J. DiGrazia, K. McKelvey, J. Bollen, and F. Rojas, "More tweets, more votes: Social media as a quantitative indicator of political behavior," PLOS ONE, vol. 8, p. e79449, Nov. 2013.

[8] M. D. Conover, B. Gonçalves, A. Flammini, and F. Menczer, "Partisan asymmetries in online political activity," EPJ Data Science, vol. 1, June 2012.

[9] T. Plotkowiak and K. Stanoevska-Slabeva, "German politicians and their twitter networks in the bundestag election 2009," First Monday, vol. 18, May 2013.

[10] G. Enli, "Twitter as arena for the authentic outsider: exploring the social media campaigns of trump and clinton in the 2016 US presidential election," European Journal of Communication, vol. 32, pp. 50-61, Feb. 2017.
[11] M. Vergeer, "Twitter and political campaigning," Sociology Compass, vol. 9, pp. 745-760, Sept. 2015.

[12] G. G. Jr, "Exame/ideia: Lula consolida liderança em 2022 e venceria bolsonaro com 45\%," URL: https://exame.com/brasil/exame-ideia-lula-consolidalideranca-para-2022-e-vence-bolsonaro-com-45/. (accessed: 09.17.2021).

[13] A. Alsaeedi and M. Z. Khan, "A study on sentiment analysis techniques of twitter data," International Journal of Advanced Computer Science and Applications, vol. 10, no. 2, pp. 361-374, 2019.

[14] R. K. Bakshi, N. Kaur, R. Kaur, and G. Kaur, "Opinion mining and sentiment analysis," in 2016 3rd International Conference on Computing for Sustainable Global Development (INDIACom), pp. 452-455, 2016.

[15] B. Liu, "Sentiment analysis and opinion mining," Synthesis lectures on human language technologies, vol. 5, no. 1, pp. 1-167, 2012.

[16] S.-M. Kim and E. Hovy, "Automatic identification of pro and con reasons in online reviews," in Proceedings of the COLING/ACL 2006 Main Conference Poster Sessions, (Sydney, Australia), pp. 483-490, Association for Computational Linguistics, July 2006.

[17] K. Sailunaz and R. Alhajj, "Emotion and sentiment analysis from twitter text," Journal of Computational Science, vol. 36, p. 101003, 2019.

[18] A. D. Dubey, “Twitter Sentiment Analysis during COVID19 Outbreak," SSRN Electronic Journal, 2020.

[19] R. J. Medford, S. N. Saleh, A. Sumarsono, T. M. Perl, and C. U. Lehmann, "An " Infodemic ": Leveraging High-Volume Twitter Data to Understand Public Sentiment for the COVID-19 Outbreak," preprint, Health Informatics, Apr. 2020.

[20] K. Garcia and L. Berton, "Topic detection and sentiment analysis in twitter content related to covid-19 from brazil and the usa," Applied Soft Computing, vol. 101, p. 107057, 2021.

[21] M. Kaur, R. Verma, and F. N. K. Otoo, "Emotions in leader's crisis communication: Twitter sentiment analysis during covid-19 outbreak," Journal of Human Behavior in the Social Environment, vol. 31, no. 1-4, pp. 362-372, 2021.

[22] I. AlAgha and O. Dahrooj, "MULTI-LEVEL ANALYSIS OF POLITICAL SENTIMENTS USING TWITTER DATA: A CASE STUDY OF THE PALESTINIAN-ISRAELI CONFLICT," Jordanian Journal of Computers and Information Technology, no. 0 , p. 1, 2019.

[23] J. Roesslein, “Tweepy: Twitter for python!," URL: https://github.com/tweepy/tweepy, 2020.

[24] C. Camille and P. Beatriz, "Internações por covid-19 duram, em média, 22 dias, aponta pesquisa," URL:https://www.cnnbrasil.com.br/saude/ 2021/03/15/internacoes-por-covid-19-duram-em-media22-dias-aponta-pesquisa. (accessed: 06.10.2021).

[25] C. Hutto and E. Gilbert, "Vader: A parsimonious rule-based model for sentiment analysis of social media text," in Proceedings of the International AAAI Conference on Web and Social Media, vol. 8, 2014.

[26] D. A. Pereira, "A survey of sentiment analysis in the portuguese language," Artificial Intelligence Review, vol. 54, no. 2, pp. 1087-1115, 2021. 
[27] S. Loria, "textblob documentation," Release 0.15, vol. 2, 2018.

[28] A. Agarwal, B. Xie, I. Vovsha, O. Rambow, and R. Passonneau, "Sentiment analysis of Twitter data," in Proceedings of the Workshop on Language in Social Media (LSM 2011), (Portland, Oregon), pp. 30-38, Association for Computational Linguistics, June 2011.

[29] S. Akhtar, D. Ghosal, A. Ekbal, P. Bhattacharyya, and S. Kurohashi, "All-in-One: Emotion, Sentiment and Intensity Prediction using a Multi-task Ensemble Framework," IEEE Transactions on Affective Computing, pp. 1-1, 2020.

[30] L.-C. Yu, J. Wang, K. R. Lai, and X. Zhang, "Refining word embeddings using intensity scores for sentimen analysis," IEEE/ACM Transactions on Audio, Speech, and Language Processing, vol. 26, no. 3, pp. 671-681, 2017.

[31] H. A. M. Voorveld, G. van Noort, D. G. Muntinga, and F. Bronner, "Engagement with social media and social media advertising: The differentiating role of platform type," Journal of Advertising, vol. 47, no. 1, pp. 38-54, 2018.

[32] C. Fernández-Villanueva and G. Bayarri-Toscano, "Legitimation of hate and political violence through memetic images: the bolsonaro campaign," Communication \& Society, pp. 449-468, 2021.

[33] C. S. Paschoal, "O novo tom axiológico da expressão "cidadão de bem": Refrações semânticas e indícios de estratificação da sociedade brasileira," Revista Memento, vol. 11, no. 1, 2020.

[34] J. Devlin, M.-W. Chang, K. Lee, and K. Toutanova, "Bert: Pre-training of deep bidirectional transformers for language understanding," arXiv preprint arXiv:1810.04805, 2018 